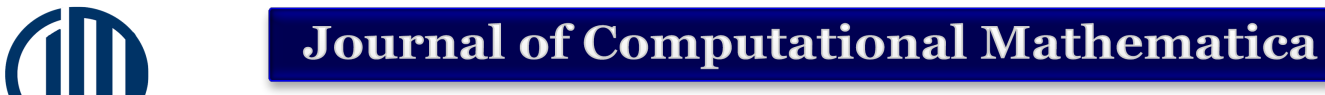 \\ Open Access
}

\section{A Study On Some Infinite Server Queues In Discrete-Time}

Syed Tahir Hussainy ${ }^{1}$, Lokesh $\mathrm{D}^{2}$

${ }^{1}$ Associate professor, Department of Mathematics, Islamiah College (Autonomous)

Vaniyambadi 635 752, Tirupattur District, Tamil Nadu India.

${ }^{2}$ Research scholar, Department of Mathematics, Islamiah College (Autonomous)

Vaniyambadi 635 752, Tirupattur District, Tamil Nadu India.

\begin{abstract}
This work analysis some discrete-time queueing mechanisms with infinitely many servers. By using a shot noise process, general results on the system size in discrete-time are given both in transient state and in steady state. For this we use the classical differentiation formula of Fá di Bruno. First two moments of the system size and distribution of the busy period of the system are also computed.
\end{abstract}

Key words: Discrete-Time Infinite Server, Shot Noise

AMS classification:

\section{Section}

Followed by the work of Meisling(1958), many authors (see for example Dafermos and Neuts (1971), Chaudhry and Templeton (1983) have considered single server discrete-time queueing systems. In the case of infinite server queues no such results are available to our knowledge. Here our objective is to study an infinite server queue in discrete-time. The number of busy servers or the number of customers in the system in transient state can be modelled as a shot noise process which is the superposition of shot effects caused by the arrivals at random epochs. This is used in queueing theory by Takács (1958) in the study of $G I / G / \infty$ system. In discrete-time queueing systems, time is considered as a discrete random variable and the events occur only at definite time points called "time marks" by Meisling. Thus arrivals occur only at these time marks which are assumed to be regularly spaced and service is initiated 
at one of these time marks and completed at another time mark. Such systems may be found in missile bases which fire at oncoming airplanes at some what regularly spaced intervals of time; in the models of storage and inventories and in the theories of reservoirs and dams. The models are explained in the next section and there we obtain the system size probabilities using the Fá di Bruno's classical differentiation formula. The mean number of busy servers and its variance at arbitrary epochs are also found. We shall use the following notations:

$D_{D}$ : Deterministic in discrete-time queueing systems

$M_{D}$ : Geometric interarrival time or service time

\section{The System $M_{D} / M_{D} / \infty$}

\section{The Model and Main Results}

This system is based on the following assumptions.

1. The time axis is divided into a succesion of intervals each of length $\Delta t$ (without loss of generality we may assume $\Delta t=1$ ). The time points which seperate these intervals are called 'time marks'. At any given time mark only one customer can arrive and more than one service completion may occur.

2. The probability of a customer arriving at a time mark is $p$ and that of no customer arrival is $q=1-p$ and the arrival of a customer at any time mark is independent of the arrival of a customer at any other time marks.

3. The service completion at any given time mark is according to a geometric distribution with probability mass function $(1-d)^{k} d, k=0,1,2, \ldots ; 0<d<1$

4. There are infinite number of servers; customers arrive from an infinite source.

The number of busy servers $N_{k}$ (which is the same as the number of customers in the system) at epoch $k$ is given by the following shot noise process:

$$
N_{k}=\sum_{i=0}^{k} I_{i, k}, k \geq 0, \text { where }
$$

\footnotetext{
${ }^{1 *}$ small1maths@gmail.com, ${ }^{2}$ loky2401@gmail.com, ${ }^{3}$ author3@gmail.com
}

Page 35 of 43 
ISSN: 2456-8686, Volume 4, Issue 2, 2020:34-43

DOI : http://doi.org/10.26524/cm78

$I_{i, k}=\left\{\begin{array}{ll}1, & \begin{array}{l}\text { if the arrival that takes place at } i \\ \text { remains in the system beyond epoch } k .\end{array} \\ 0, & \begin{array}{l}\text { if there is no arrival or the unit arrived at } i \\ \text { completes its service at or before } k .\end{array}\end{array}\right.$ Then

$$
\begin{aligned}
& \operatorname{Pr}\left\{I_{i, k}=1\right\}=p(1-d)^{k-i}, \text { and } \\
& \operatorname{Pr}\left\{I_{i, k}=0\right\}=p \sum_{h=0}^{k-i-1}(1-d)^{h} d+(1-p) .
\end{aligned}
$$

The probability generating function for $N_{k}$, denoted by $\phi_{k}(s)$, is then given by

$$
\phi_{k}(s)=E\left[s^{N_{k}}\right]=\prod_{i=0}^{k} E\left[s^{I_{i, k}}\right]
$$

since $I_{i, k}$ 's are independent.

We have

$$
\begin{aligned}
E\left[s^{I_{i, k}}\right] & =s p(1-d)^{k-i}+(1-p)+p d \sum_{h=0}^{k-i-1}(1-d)^{h} \\
& =\left[1+p(1-d)^{k-i}(s-1)\right]
\end{aligned}
$$

Therefore

$$
\phi_{k}(s)=\Pi_{i=0}^{k}\left[1+p(1-d)^{k-i}(s-1)\right]
$$

From this, we have

$$
\begin{aligned}
\phi_{k+1}(s) & =\phi_{k}(s) \phi_{0, k+1}(s) \text { and in general } \\
\phi_{k+n}(s) & =\phi_{k}(s) \phi_{0, k+n-1} \phi_{0, k+n-1}(s) \cdots \phi_{0, k+1}(s) \\
& =\phi_{k+n-1}(s) \phi_{0, k+n}(s), \text { where } \\
\phi_{0, k+n}(s) & =\left[1+p(1-d)^{k+n}(s-1)\right]
\end{aligned}
$$

From (1)

$$
\ln \left[\phi_{k}(s)\right]=\sum_{i=0}^{k} \ln \left[1+p(1-d)^{k-i}(s-1)\right]
$$

Taking the logarithmic expansion of the right hand side of (3) and collecting the terms, we get

$$
\left.\phi_{k}(s)=\exp \left\{\sum_{j=1}^{\infty}(-1)^{j+1}[p(s-1)]^{j} / j\right]\left[\left(1-(1-d)^{j(k+1)}\right) /\left(1-(1-d)^{j}\right)\right]\right\}
$$


In the limiting case

$$
\left.\phi(s)=\lim _{k \rightarrow \infty} \phi_{k}(s)=\exp \left\{\sum_{j=1}^{\infty}(-1)^{j+1}[p(s-1)]^{j} / j\left(1-(1-d)^{j}\right)\right]\right\}
$$

From (2),

$$
\lim _{k \rightarrow \infty} \phi_{k+n}(s)=\lim _{k \rightarrow \infty} \phi_{k}(s) \cdot 1=\phi(s)
$$

To obtain the system size probabilities at any arbitrary epoch, we use the Fá di Bruno's differentiation formula (see, Klimo and Neuts (1973)), which is given below. Fáa di Bruno's formula. Assuming the existence of all derivatives involved,

$$
\begin{gathered}
\left\{D^{n}[f(\phi(x))]\right\}_{x=0}=\sum_{r=1}^{n}\left\{D^{r}[f(y)]\right\}_{y=\phi(0)} \times \sum_{\substack{j_{1}+\ldots+j_{n}=n \\
j_{1}+2 j_{2}+\cdots+\cdots n_{n}=r \\
j_{1} \geq 0, \cdots, j_{n} \geq 0}}\left(n ! / j_{1} ! \cdots j_{n} !\right) \times \\
\left(\phi^{1}(0) / 1 !\right)^{j_{1}}\left(\phi^{2}(0) / 2 !\right)^{j_{2}} \cdots\left(\phi^{n}(0) / n !\right)^{j_{n}}
\end{gathered}
$$

where $D^{n}$ stands for the $n$-th derivative of $f(\cdot)$ and $\phi^{n}(\cdot)$ denote the $n$-th derivative of the function $\phi(\cdot)$. Probability that the number of busy servers (that is the number of customers in the system) at epoch $k$ is $(0 \leq l \leq k)$ denoted by $P_{k}(l)$, is

$$
(1 / l !)\left\{D^{l}\left[\phi_{k}(s)\right]\right\}_{s=0}
$$

Write $q(s)=\sum_{j=1}^{\infty}(-1)^{j+1}[p(s-1) / j]^{j}\left[\left(1-(1-d)^{j(k+1)}\right) / 1-(1-d)^{j}\right]$. Using the formula (5)

$$
\begin{gathered}
\left\{D^{l}(\phi(k)(s))\right\}_{s=0}=\sum_{r=1}^{l}\left\{D^{r}\left(e^{y}\right)\right\}_{y=q(0)} \sum_{\substack{j_{1}+\ldots+j_{l}=r \\
j_{1}+2 j_{2}+\ldots+l_{l}=l \\
j_{1} \geq 0, \cdots, j_{l} \geq 0}}\left(l ! / j_{1} ! \cdots j_{l} !\right) \times \\
\left(q^{1}(0) / 1 !\right)^{j_{1}}\left(q^{2}(0) / 2 !\right)^{j_{2}} \cdots(q(0) / l !)^{j_{l}}
\end{gathered}
$$

where $q^{i}(0)$ is the $i$-th derivative of $q(s)$ at $s=0$.

Therefore 


$$
\begin{aligned}
& P_{k}(l)=(1 / l !) \sum_{r=1}^{l} \exp \left\{\sum_{j=1}^{\infty}(-1)^{2 j+1}\left(p^{j} / j\right)\left[\frac{\left(1-(1-d)^{j(k+1)}\right)}{\left(1-(1-d)^{j}\right)}\right]\right\} \\
& \times \sum_{\substack{j_{1}+\cdots+j_{l}=r \\
j_{1}+2 j_{2}+\ldots+l j_{l}=l \\
j_{1} \geq 0, \cdots, j_{l} \geq 0}}\left(l ! / j_{1} ! \cdots j_{l} !\right) \times\left\{\sum_{j=1}^{\infty}(-1)^{2 j} p^{j} \frac{\left(1-(1-d)^{j(k+1)}\right)}{\left(1-(1-d)^{j}\right.}\right\}^{j_{1}} \\
& \times \cdots \times\left\{\sum_{j=l}^{\infty}(-1)^{2 j+1} p^{j}(j-1) \cdots(j-l+1) \frac{\left(l-(1-d)^{j(k+1)}\right)}{\left(1-(1-d)^{j}\right)}\right\}^{j_{l}} \\
& P(l)=\lim _{k \rightarrow \infty} P k(l) \\
& =(1 / l !) \sum_{r=1}^{1} \exp \left\{\sum_{j=1}^{\infty}(-1)^{2 j+1}\left[p^{j} / j\left(1-(1-d)^{j}\right)\right]\right\} \\
& \sum_{\substack{j_{1}+\cdots+j_{l}=r \\
j_{1}+2 j_{2}+\ldots+l j_{l}=l \\
j_{1} \geq 0, \cdots, j_{l} \geq 0}}\left\{\sum_{j=1}^{\infty}(-1)^{2 j} p^{j} / 1-(1-d)^{j}\right\}^{j_{i}} \cdots \\
& \left\{\sum_{j=l}^{\infty}(-1)^{2 j-l+1} p^{j}(j-1) \cdots(j-l+1) /\left(1-(1-d)^{j}\right)\right\}^{j l}
\end{aligned}
$$

\section{The Moments}

The first two moments for $N_{k}$ can be computed directly from (1). Differentiating (4) with respect to $s$ and set $s=1$, we get the expected number of busy servers, $E\left(N_{k}\right)$. That is,

$$
E\left(N_{k}\right)=\phi_{k}^{\prime}(1)=\left[p\left(1-(1-d)^{k+1}\right) / d\right]
$$

Differentiating (3) twice and setting $s=1$, we get

$$
\phi_{k}^{2}(1)=\left[p^{2}\left(1-(1-d)^{2 k+2}\right) / d(d-2)\right]+\left[\phi_{k}^{\prime}(1)\right]^{2}
$$

Therefore, variance of $N_{k}$ is

$$
\operatorname{Var}\left(N_{k}\right)=\left[p^{2}\left(1-(1-d)^{2 k+2}\right) / d(d-2)\right]+\left(p\left(1-(1-d)^{k+1}\right) / d\right)
$$

From (6) and (7), $E(N)=\lim _{k \rightarrow \infty} E(N k)=\frac{p}{d}$, which coincides with the continuous time result for the $M / M / \infty$ system and

$$
\operatorname{Var}(N)=\lim _{k \rightarrow \infty} \operatorname{Var}(N k)=E(N)[1+p /(d-2)]
$$


ISSN: 2456-8686, Volume 4, Issue 2, 2020:34-43

DOI : http://doi.org/10.26524/cm78

\section{THE SYSTEM $D_{D} / M_{D} / \infty$}

\section{The Model}

In this model the interarrival time distribution is assumed to be deterministic $\left(D_{D}\right)$. That is if $A$ is the time between two consecutive arrivals of customers, then $\operatorname{Pr}$ (the interarrival time $=A)=1$. Here we assume $A=1$. The service time distribution is assumed to be geometric and all other assumptions are same as in the previous case. The number of busy servers or the number of customers in the system at epoch $k$ is given by the following shot noise process:

$$
\begin{aligned}
& N_{k}=\sum_{i=0}^{k} I_{i, k}, \text { where } \\
& I_{i, k}= \begin{cases}1, & \text { if the unit arriving at epoch } i \\
& \text { remains in the system beyond } k \\
0, & \text { otherwise. }\end{cases}
\end{aligned}
$$

Now

$$
\begin{aligned}
& \operatorname{Pr}\left\{I_{i, k}=1\right\}=(1-d)^{k-i} \text { and } \\
& \operatorname{Pr}\left\{I_{i, k}=0\right\}=\sum_{h=0}^{k-i-1}(1-d)^{h} d
\end{aligned}
$$

The probability generating function for $N_{k}$ is

$$
\phi_{k}(s)=E\left[s^{N_{k}}\right]=\prod_{i=0}^{k} E\left[s^{I_{i, k}}\right], \text { since } I_{i, k}{ }^{\prime} \mathrm{s}
$$

are independent. Therefore

$$
\phi_{k}(s)=\Pi_{i=0}^{k}\left[1+(1-d)^{k-i}(s-1)\right]
$$

Equation (1) and (8) are same when $p=1$, as is expected. As in the previous case, we have

$$
\begin{aligned}
\phi_{k+n}(s) & =\phi_{k+n-1}(s) \phi_{0, k+n}(s), \text { where } \\
\phi_{0, k+n}(s) & =\left[1+(1-d)^{k+n}(s-1)\right]
\end{aligned}
$$

From (8)

$$
\ln \phi_{k}(s)=\sum_{i=0}^{k} \ln \left[1+(1-d)^{k-i}(s-1)\right]
$$


ISSN: 2456-8686, Volume 4, Issue 2, 2020:34-43

DOI : http://doi.org/10.26524/cm78

Repeating the same procedure as in the previous model, we get

$$
\phi_{k}(s)=\exp \left\{\sum_{j=1}^{\infty}(-1)^{j+1}\left[(s-1)^{j} / j\right]\left[\frac{1-(1-d)^{j(k+1)}}{\left(1-(1-d)^{j}\right)}\right]\right\}
$$

In the limiting case

$$
\begin{aligned}
\phi(s) & =\lim _{k \rightarrow \infty} \phi k(s) \\
& =\exp \left\{\sum_{j=1}(-1)^{j+1}\left((s-1)^{j}\right) / j\left(1-(1-d)^{j}\right)\right\}
\end{aligned}
$$

As in the above model, probability that there are $l(0 \leq l \leq k)$ busy servers at epoch $k$ is $P_{k}(l)$ which is given by

$$
\begin{aligned}
& P_{k}(l)=(1 / l !) \sum_{r=1}^{l} \exp \left\{\sum_{j=1}^{\infty} \frac{\left[(-1)^{2 j+1}\left(1-(1-d)^{j(k+1)}\right)\right]}{\left[j\left(1-(1-d)^{j}\right)\right]}\right\} \\
& \times \sum_{\substack{j_{1}+\cdots+j_{l}=r \\
j_{1}+2 j_{2}+\ldots+l j_{l}=l \\
j_{1} \geq 0, \cdots, j_{l} \geq 0}}\left(l ! / j_{1} ! \cdots j_{i} !\right) \times \\
& \times\left\{\sum_{j=1}^{\infty}(-1)^{2 j} \frac{\left(1-(1-d)^{j(k+1)}\right)}{\left(1-(1-d)^{j}\right)}\right\}^{j_{1}} \\
& \times \cdots \times\left\{(-1)^{2 j-l+1}(j-1)(j-2) \cdots(j-l+1) \frac{\left(l-(1-d)^{j(k+1)}\right)}{\left(1-(1-d)^{j}\right)}\right\}^{j_{l}} \\
& p(l)=\lim _{k \rightarrow \infty} p_{k}(l) \\
& =(1 / l !) \sum_{r=1}^{l} \exp \left\{\sum_{j=1}^{\infty}\left[\frac{(-1)^{2 j+1}}{j\left(1-(1-d)^{j}\right)}\right]\right\} \\
& \sum_{\substack{j_{1}+\cdots+j_{l}=r \\
j_{1}+2 j_{2}+\ldots+l j_{l}=l \\
j_{1} \geq 0, \cdots, j_{l} \geq 0}}\left(l ! / j_{1} ! \cdots j_{l} !\right)\left\{\sum_{j=1}^{\infty} \frac{(-1)^{2 j}}{\left(1-(1-d)^{j}\right)}\right\}^{j_{1}} \\
& \times \cdots \times\left\{\sum_{j=1}^{\infty} \frac{\left[(-1)^{2 j-l+1}(j-1) \cdots(j-l+1)\right]}{\left(1-(1-d)^{j}\right)}\right\}^{j l}
\end{aligned}
$$

\section{The Moments}


ISSN: 2456-8686, Volume 4, Issue 2, 2020:34-43

DOI : http://doi.org/10.26524/cm78

Differentiating (8) with respect to $s$ and setting $s=1$, we get

$$
\begin{aligned}
& \phi_{k}^{1}(l)=E\left(N_{k}\right)=\left[1-(1-d)^{k+1}\right] / d \text { and } \\
& \left.\phi_{k}^{2}(l)=\left[1-(1-d)^{2 k+2}\right] / d(d-2)\right]+\left[\phi_{k}^{\prime}(l)\right]^{2}
\end{aligned}
$$

Therefore

$$
\operatorname{Var}\left[N_{k}\right]=\left[\left(1-(1-d)^{2 k+2}\right) / d(d-2)\right]+\left(1-(1-d)^{k+1}\right) / d
$$

From (11) and 12

$$
\begin{aligned}
E(N) & =\lim _{k \rightarrow \infty} E(N k)=1 / d \text { and } \\
\operatorname{Var}(N) & =\lim _{k \rightarrow \infty} \operatorname{Var}(N k)=E(N)[(d-1) /(d-2)]
\end{aligned}
$$

That is when $p=1$, the models $B_{D} / M_{D} / \infty$ and $D_{D} / M_{D} / \infty$ are the same.

\section{THE SYSTEM $M_{D} / D_{D} / \infty$}

Here the interarrival time distribution is as in $M_{D} / M_{D} / \infty$ system but the service time distribution is assumed to be deterministic $\left(D_{D}\right)$.

That is, if $S$ is the service time, then for $r \geq 1$

$$
\operatorname{Pr}(S=r)= \begin{cases}1 & \text { if } S=r \\ 0 & \text { if } S \neq r\end{cases}
$$

The number of busy servers at epoch $k$ is

$$
\begin{aligned}
& N_{k}=\sum_{i=0}^{k} I_{i, k}, \text { where } \\
& I_{i, k}= \begin{cases}1, & \text { if } k \leq i+r-1 \\
0, & \text { otherwise }\end{cases}
\end{aligned}
$$

$$
\begin{aligned}
& \operatorname{Pr}\left\{I_{i, k}=1\right\} \quad=\quad p, \text { if } k \leq i+r-1 \text { and } \\
& \operatorname{Pr}\left\{I_{i, k}=0\right\} \quad=\quad 1-p, \text { if } k>i+r-1 \text { or there is no arrival. }
\end{aligned}
$$


ISSN: 2456-8686, Volume 4, Issue 2, 2020:34-43

DOI : http://doi.org/10.26524/cm78

The generating function for $N_{k}$ is

$$
\begin{aligned}
\phi_{k}(s) & =E\left[s^{N_{k}}\right]=E\left[s^{\sum_{i=0}^{k} I_{i, k}}\right] \\
& =E\left[s^{I_{k-r+1, k}}\right] \cdots E\left[s^{I^{k, k}}\right] \\
\text { Now } E\left[S^{I^{i, k}}\right] & =p s+q, \text { where } q=1-p .
\end{aligned}
$$

Therefore $\phi_{k}(s)=(p s+q)^{r-1}$, which is the probability generating function of binomial random variable and is independent of $k$. Probability that the number of busy servers at epoch $k$ is $l(\leq r-1)$ is $P_{k}(l)$ which is the coefficient of $s^{l}$ in $\phi_{k}(s)$. Therefore

$$
P_{k}(l)=\left(\begin{array}{c}
r-1 \\
l
\end{array}\right) p^{l} q^{r-1-l}, l=0,1,2, \ldots, r-1
$$

The right hand side of $P_{k}(l)$ is independent of $k$ and hence it remains the same as $k \rightarrow \infty$. Therefore

$$
\begin{aligned}
E\left(N_{k}\right) & =E(N)=(r-1) p \text { and } \\
\operatorname{Var}\left(N_{k}\right) & =\operatorname{Var}(N)=(r-1) p q
\end{aligned}
$$

\section{Distribution Of The Busy Period Of The $M_{D} / M_{D} / \infty$ SYSTEM}

We define the busy period of the system as the time until all servers are idle starting from the service initiation at an arrival epoch. Let $B_{0,0}$ denote this random variable. Probability that an arrival at epoch ' 0 ' completes its service before the epoch $m$ is

$$
\sum_{r=0}^{m-1}(1-d)^{r} d=1-(1-d)^{m}, m>1
$$

Probability that there are $n$ arrivals prior to time $m$ is

$$
\left(\begin{array}{c}
m-1 \\
n
\end{array}\right) p^{n}(1-p)^{m-1-n}, 0 \leq n \leq m-1 .
$$

${ }^{1 *}$ small1maths@gmail.com, ${ }^{2}$ loky2401@gmail.com, ${ }^{3}$ author3@gmail.com

Page 42 of 43 
Let $0<j_{1}<j_{2}<\cdots<j_{n}<m$ these arrival epochs and let

$$
\begin{aligned}
B_{0,0}^{(m)} & =\operatorname{Pr}\left\{B_{0,0}=m\right\} \\
& =\sum_{n=0}^{m-1} \operatorname{Pr}\left\{B_{0,0}=m \mid n \text { arrivals before } m\right\}\left(\begin{array}{c}
m-1 \\
n
\end{array}\right) p^{n}(1-p)^{m-1-n} \\
& =\sum_{n=0}^{m-1} \operatorname{Pr}\left\{I_{j_{1}, m}=0, I_{j_{2}, m}=0, \cdots I_{j_{n}, m}=0\right\}\left(\begin{array}{c}
m-1 \\
n
\end{array}\right) p^{n}(1-p)^{n-1-m} \\
& =\sum_{n=0}^{m-1} \prod_{i=1}^{n} \sum_{r=0}^{m-j_{i}-1}(1-d)^{r} d\left(\begin{array}{c}
m-1 \\
n
\end{array}\right) p^{n}(1-p)^{m-1-n} \\
& =\sum_{n=0}^{m} \prod_{i=1}^{n}\left(\begin{array}{c}
m-1 \\
n
\end{array}\right) p^{n}(1-p)^{m-1-n}\left[1-(1-d)^{m-j_{i}}\right]
\end{aligned}
$$

\section{Conclusion}

In this paper, we discussed the some model and derived some important results. Also we discussed about moments. Also we discussed distribution of the busy period.

\section{References}

[1] Chaudhry ML and Templeton JGC, A First Course in Bulk Queues, John Wiley and Sons, New York(1983).

[2] Dafermos SC and Neuts MF, A single Server Queue in Discrete - Time, Cah. Cent. Etud. Resh. Oper. 13, 23-40(1971).

[3] Klimko M and Neuts MF, The single Server Queue in Discrete Time - Numerical Analysis II, Nav. Res. Logist. 20,2,305-319.

[4] Liu L, Kashyap BRK and Templeton JGC, On the System $G I^{X} / G / \infty, J$. Appl. Prob. 27,671.683.(1990).

[5] Meisling T, Discrete-Time Queueing Theory, Oper. Res. 6 , 96-105(1958).

[6] Takács, L, On a Coincidence Problem in Telephone Traffic, Act. Math. Acad. Sci Hung, 9,45-80(1958). 